\title{
Publisher Correction: Asteroids: Insights on the olivine mystery
}

Luca Maltagliati

Correction to: Nature Astronomy https://doi.org/10.1038/s41550-019-0704-4, published online 30 January 2019.

In the version of this Research Highlight originally published, it was mistakenly stated that DeMeo et al. performed follow-up observations using the Mauna Kea telescope. These observations were actually performed using NASA's Infrared Telescope Facility at the Mauna Kea Observatory. The last sentence of the first paragraph has now been corrected to read: 'Francesca DeMeo and collaborators performed follow-up spectral observations of A-type candidates with NASA's Infrared Telescope Facility and the 6.5-m Magellan telescope in order to obtain a better characterization of olivine asteroids in the main belt.'

Published online: 11 February 2019

https://doi.org/10.1038/s41550-019-0717-z 